\title{
A STUDY OF A PULSAR WIND DRIVEN STRUCTURE IN FAR-INFRARED IRAS MAP AT LATTITUDE -10 ${ }^{\circ}$
}

\section{Ajay Kumar Jha and Binil Aryal}

Journal of Institute of Science and Technology

Volume 22, Issue 1, July 2017

ISSN: 2469-9062 (print), 2467-9240 (e)

\section{Editors:}

Prof. Dr. Kumar Sapkota

Prof. Dr. Armila Rajbhandari

Assoc. Prof. Dr. Gopi Chandra Kaphle

JIST, 22 (1): 1-9 (2017)

Published by:

Institute of Science and Technology

Tribhuvan University

Kirtipur, Kathmandu, Nepal 


\title{
A STUDY OF A PULSAR WIND DRIVEN STRUCTURE IN FAR-INFRARED IRAS MAP AT LATTITUDE -10
}

\author{
Ajay Kumar Jha* and Binil Aryal \\ Central Department of Physics, Tribhuvan University, Kirtipur, Nepal \\ Corresponding E-mail: astroajay123@gmail.com
}

\begin{abstract}
A systematic search of dust structure in the far infrared $(100 \mu \mathrm{m}$ and $60 \mu \mathrm{m})$ IRAS (Infrared Astronomical Satellite) survey was performed using Sky View Observatory. In order to find the possible candidate, we used SIMBAD database to locate discrete sources in the region. A deep cavity-like isolated far infrared dust structure (size $\sim 4.46 \mathrm{pc} \times 2.23 \mathrm{pc}$ ) at galactic longitude: $284.360^{\circ}$, galactic latitude: $-9.549^{\circ}$ was found at the distance of about $375 \mathrm{pc}$. We have studied the flux density variation and then calculated temperature and mass profile of the dust and excess mass using data reduction software ALADIN 7.5 within this region. The dust color temperature was found to lie in the range $23.40 \mathrm{~K}$ to $29.28 \mathrm{~K}$. An offset temperature of about 6.0 $\mathrm{K}$ was found. The total mass of the dust structure was found to be about $2.55 \times 10^{27} \mathrm{~kg}$ and the excess mass per pixel was $2.52 \times 10^{24} \mathrm{~kg}$. We also studied the rate of mass loading around the structure. The energy of the pulsar required to create that inhomogeneity in the structure was calculated to be $5.04 \times 10^{36} \mathrm{~J}$. Possible explanations of results will be presented.
\end{abstract}

Keywords: IRAS survey, Flux density, ALADIN 7.5, Pulsar wind, Galactic parameter.

\section{INTRODUCTION}

Massive star (8-25 $\left.\mathrm{M}_{\odot}\right)$ ends its life in a supernova explosion and center of star collapse to form neutron star. Materials (stellar debris) around it move away with very high rate of speed. This event not only produces a disturbance in interstellar medium (ISM) but in other hand it has enriched the medium with material heavier than hydrogen and helium (Karttunen et al., 2007).

Because of conservation of angular momentum neutron star spins very rapidly. There is magnetic field that was in the original star that gets compressed and frozen into that surface of neutron star. So neutron stars have strong spin magnet in space. The charge particle of Neutron star move at relativistic speed varying the speed of light and that cause sorts of radiation be generated. Radio waves come out from the region between the magnetic axis and spinning axis of neutron stars as their spinning and magnetic axes don't align to each other just like our earth. If these radio beams pass by earth through our line of sight, we see pulse of radio wave. So, because of these sweeping of light house type beam across our line of sight we see pulses of radiation that's why we called them pulsars (Bhattacharya et al., 2008).

Hence, Pulsars are rotating neutron stars in which co-rotating magnetic field $\left(\sim 10^{12} \mathrm{G}\right)$ can accelerate charge particle to the cosmic ray energies. These rapidly rotating neutron stars have been proposed as the energy source in supernova remnant by Wheeler and Pacini in 1966. Further in 1968, Gold and Pacini have suggested that pulsars are rotating magnetic neutron stars formed in supernova explosion and after the explosion they dashing away envelop of supernova remnant at the speed of $100-1000 \mathrm{~km} / \mathrm{Sec}$ (Pacini, 1968).

Pulsar moves through either ISM or in more interestingly in supernova remnant. In both cases, there exists a three folds structure consisting of the wind. These three fold structures are i) Terminating Shock, ii) Contact Discontinuity, and iii) Bow Shock.

Pulsar winds are composed of charged particles accelerated to relativistic speed by the rapidly rotating, super strong magnetic field of the spinning pulsar. The pulsar wind streams in the ISM, creating a standing shock wave, where it is decelerated to sub-relativistic speed. As, Pulsars are thought to eject relativistic moving charge particles that energies the surrounding medium with the formation of pulsar wind and thus formed a pulsar wind driven structure in ISM (Becker, 2009).

The pulsar wind driven structure also known as cavity is the very low temperature and mass region so we need that spectrum through which the region 
become more visible and we can get more information which is actually the region of Infrared from electromagnetic spectrum. In cavity we studied old and very cold dusts that are totally opaque in the optical but bright in the Infrared Region. There are many space telescopes studying in Infrared Region. We took data provided by IRAS (Infrared Astronomical Satellite). The IRAS mission was major advance for astronomy. Almost the entire sky was covered in four pass bands (12, 25, 60 and 100 micron). Although the IRAS mission took place two decades ago - the maps are still not exhausted of their riches which is one of the reason that motivated us to carry our study in IRAS map and discovered the cavity structure around pulsar (Beichman et al.,1988).

\section{Region of Interest}

Sky view Virtual Observatory was used in order to search a deep cavity structure for different wavelength IRAS cbands. We carried a systematic search of IRAS maps available in the Sky view Virtual Observatory from April 2014 to October 2014. Skyview is intended as a quick look facility to see our sky in different spectrum. Skyview is not exactly a telescope but the collection of large number of database of astronomical surveys that simulate the images taken by telescopes or satellites. The core of Sky view is a geometry engine which enables the transformation of the data from existing surveys into the image we have requested. We downloaded the JPEG image of about 100 pulsars and we took some image for the further study. Out of the selected candidates, we selected one image for systematic research by finding the work that has been done on the objects near to our candidate with the help of SIMBAD.

In the process of scanning the sky covering the range of J2000 coordinate system provided by Suh
\& Kwon (2009), we found some interesting isolated structures surrounding planetary nebula, pulsars, white dwarfs and different galaxies. We entered 100 Pulsar coordinates of 1428 given from catalog in terms of R.A. (hour, min, sec) and Dec (degree, min, sec) and downloaded 800 different images in JPEG format (200 images each in four different bands of IRAS; $12 \mu \mathrm{m}, 25 \mu \mathrm{m}, 60 \mu \mathrm{m}$ and $100 \mu \mathrm{m}$ ) of size $500 \times 500$ pixels $($ Suh \& Kwon, 2009).

For the proper study of the isolated cavity like structure, its emission in $100 \mu \mathrm{m}$ and $60 \mu \mathrm{m}$ is essential. Also it should not have any connection with larger molecular cloud present nearby. Hence using these criteria we selected only few images for further study. The selected images have prominent emission in all four bands and are isolated from their nearby structure. Also SIMBAD was used to find the degree of study made on these individual structures. We found the isolated cavity like structure having size $\sim 1^{\circ}$ found in Galactic longitude RA $(\mathrm{J} 2000)=18^{\mathrm{h}} 57^{\mathrm{m}} 26.45^{\mathrm{s}}$ and Galactic latitude Dec $(\mathrm{J} 2000)=-10^{\circ} 27^{\prime} 01^{\prime}$ ' was less studied and hence making it more appropriate for our study. In addition, the selected preferred cavity is taken nearly rounded or symmetrical in shape. These structures have been looked critically. Finally best structure in which the flux density has minimum value in the cavity has selected for further study. Figure 1a and $\mathrm{b}$ show 100 and 60 micron image of selected dust structure. The location of Pulsar stars in Figure $1 \mathrm{a}$ and $\mathrm{b}$ are RA $(\mathrm{J} 2000)=18^{\mathrm{h}} 57^{\mathrm{m}} 26.45^{\mathrm{s}}$, $\operatorname{Dec}(\mathrm{J} 2000)=-10^{\circ} 27^{\prime} 01^{\prime}$ '. In the structure, minimum flux density is found at RA $(\mathrm{J} 2000)=$ $18^{\mathrm{h}} 57^{\mathrm{m}} 53.81^{\mathrm{s}}, \quad$ Dec $(\mathrm{J} 2000)=-10^{\circ} 29^{\prime} 05.1^{\prime \prime}$. The distance to this structure is $375 \mathrm{pc}$ which has been estimated by using star-count method and colorcolor/color-magnitude diagram (Giles, 1982).

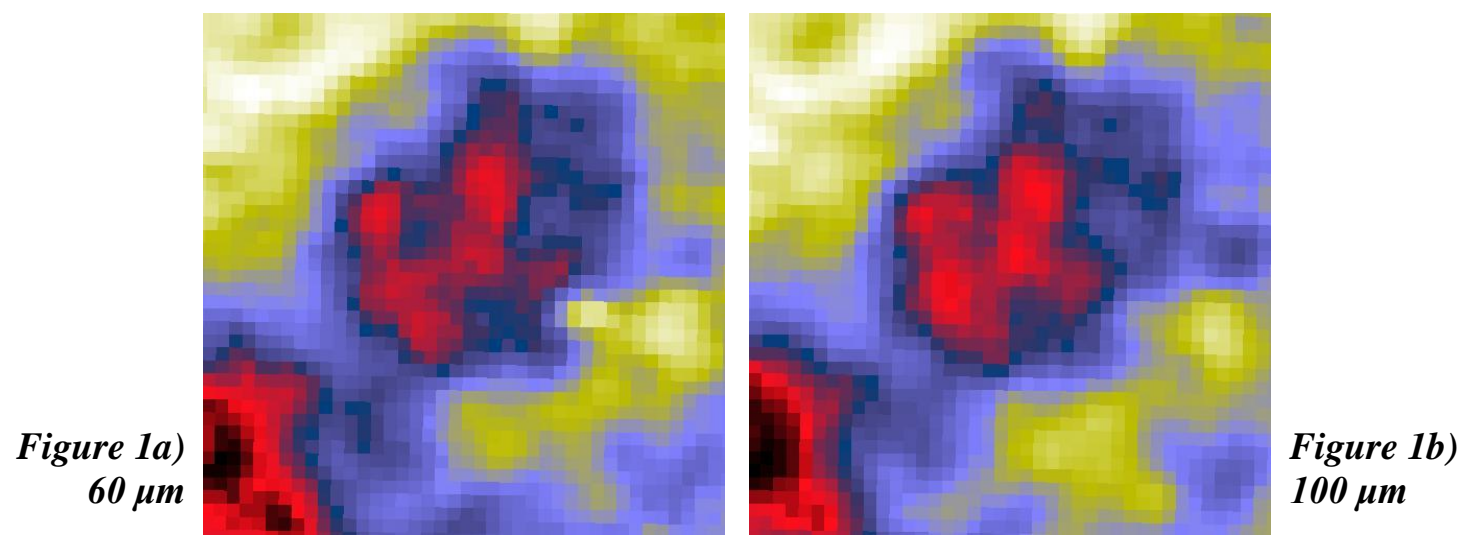

Fig. $1.1^{\circ} \times 1^{\circ}$ IRAS image of our region of interest in $60 \mu \mathrm{m}$, and $100 \mu \mathrm{m}$ bands. In all bands, fine cavity including Pulsar RA/Dec(J2000) $=18^{h} 57^{m} 26.45^{s} /-10^{\circ} 27^{\prime}$ 01'’(SkyView, 2014). 


\section{MATERIALS AND METHODS}

We adopted the following methods to find the size, dust color temperature and dust mass of the structure. In addition, we calculated the excess mass and the energy of the pulsar wind needed to expel the excess mass.

\section{Dust Color temperature Estimation}

The dust temperature $T_{d}$ in each pixel of a FITS image can be obtained by assuming that the dust in a single beam is isothermal and that the observed ratio of 60 micron to 100 micron emission is due to black body radiation from dust grains at $T_{d}$, modified by a power law emissivity spectral index. The flux density $F_{i}$ of emission at a wavelength $\lambda_{i}$, is given by,

$F_{i}=\left[\frac{2 h c}{\lambda_{i}^{3}\left(e^{\frac{h c}{\lambda_{i} \mathrm{KT}_{d}}}-1\right)}\right] N_{d} \alpha \lambda_{i}^{-\beta} \Omega_{i}$

Where $\mathrm{N}_{\mathrm{d}}$ represents column density of dust grains, $\alpha$ is a constant that relates the flux to the optical depth of the dust, $\beta$ is the emissivity spectral index and $\Omega_{\mathrm{i}}$ is the solid angle subtended at wavelengths $\left(\lambda_{\mathrm{i})}\right.$ by the detector.

Following Dupac et al. (2003), we used the equation,

$\beta=\frac{1}{\delta+\omega \mathrm{T}_{\mathrm{d}}}$

To describe the observed inverse relationship between temperature and emissivity spectral index. Where $\delta$ and $\omega$ are free parameters and are derived separately for each cloud.

With the assumptions that the dust emission is optically thin at 60 micron and 100 micron and that $\Omega_{60} \approx \Omega_{100}$ (true for IRAS image), we can write the ratio, $R$, of the flux densities at 60 micron and 100 micron as

$R=0.6^{-(3+\beta)} \frac{{\frac{1}{T_{d}}}^{\frac{144}{T^{2}}}}{\mathrm{e}^{\frac{240}{T_{d}}}-1}$

For a smaller value of $T_{d}, 1$ can be dropped from both numerator and denominator of Eq. (3) and it takes the form

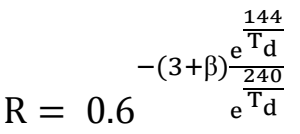

Taking natural logarithm on both sides of Eq. (4) we find the expression for the temperature as

$T_{d}=-96 \frac{1}{\ln \left\{R \times 0.6^{(3+\beta)}\right\}}$

The value of the spectral emissivity index $(\beta)$ depends on dust grain properties as composition, size, and compactness. For reference, a pure blackbody would have $\beta=0$, the amorphous layerlattice matter has $\beta \sim 1$, and the metals and crystalline dielectrics have $\beta \sim 2$. ( Dupac et al. 2003)

Where $\mathrm{R}$ is given by (Schnee et al., 2005)

$\mathrm{R}=\frac{\mathrm{F}(60 \mu \mathrm{m})}{\mathrm{F}(100 \mu \mathrm{m})}$

$\mathrm{F}$ (60 micron) and $\mathrm{F}(100$ micron) are the flux densities at $60 \mu \mathrm{m}$ and $100 \mu \mathrm{m}$, respectively. In this way we can use equation (5) for the determination of the dust grain temperature (Dupac et al. 2003).

\section{Dust Mass Estimation}

The dust masses are estimated from the IR flux densities. The resulting dust mass depends on the physical and chemical properties of the dust grains, the adopted dust temperature $T_{d}$ and the distance $D$ to the object (Hildebrand, 1983).

$$
M_{d u s t}=\frac{4}{3} \frac{a \rho}{Q_{v}}\left[\frac{S_{v} D^{2}}{B(v, T)}\right]
$$

Where, $a=$ Weighted grain size $=0.1 \mu \mathrm{m}$

$\rho=$ Grain density $=3000 \mathrm{Kg} \mathrm{m}^{-3}$

$Q_{\nu}=$ grain emissivity $=0.0010$ for $100 \mu \mathrm{m}$ and 0.0046 for $60 \mu \mathrm{m}$ respectively (Young et al., 1993)

$S_{v}=$ total flux density of the region whose mass is to be determined

$S_{v}=f \times 5.288 \times 10^{-9} \mathrm{MJy} / \mathrm{Sr}$

$D=$ distance of the structure

$B(v, T)=$ Planck's function

$B(v, T)=\frac{2 h v^{3}}{c^{2}}\left[\frac{1}{e^{\frac{h v}{k T}}-1}\right]$ 
Where,

$h=$ Planck's constant

$c=$ velocity of light

$v=$ frequency at which the emission is observed

$T=$ the average temperatures of the region.

Values of different parameters we used in the calculation of the dust mass in our interest are as follows:

Using these values the equation (7) takes the form

$$
M_{d u s t}=0.4\left[\frac{S_{v} D^{2}}{B(v, T)}\right]
$$

\section{Energy Estimation}

To estimate the energy of the region of the interest we assume here that our targeted energy values are those with this energy value mass of central region is moving outward. The outer wind expands quietly at a certain velocity (Johnston et al., 2001). This is by far the largest part of the wind (extending up to $1000-100000 R_{\star}$, Star Radius, depending on the mass-loss history) with temperatures decreasing to $\sim 10 \mathrm{~K}$. Chemically, the outer wind remains quite active with interstellar UV photons photo dissociating molecules. The products of photo dissociation can trigger subsequent molecule formation through neutral-neutral and ion-molecule reactions. We use calculated value of average mass deficit per pixel. With the help of classical kinetic energy relation we are focused to estimate the values of outflow energy.

$$
E=\frac{1}{2} M_{\text {deficit }} u_{\text {out }}^{2}
$$

$M_{\text {deficit }}$ values are obtained from subtraction between projected mass and real mass of small (inner) circle of our cavity like structure.

\section{RESULTS AND DISCUSSION}

Figure 2 shows dust structures of the selected cavity at 100 microns. The contour levels are drawn. Optimum isocontours are found at levels 20, 46 and 79 respectively. The lines $\mathrm{AB} \& \mathrm{CD}$ represent the major and minor diameters passing through minimum flux density (flux-minima). The line $\mathrm{EF}$ is the line joining minimum flux and maximum temperature.

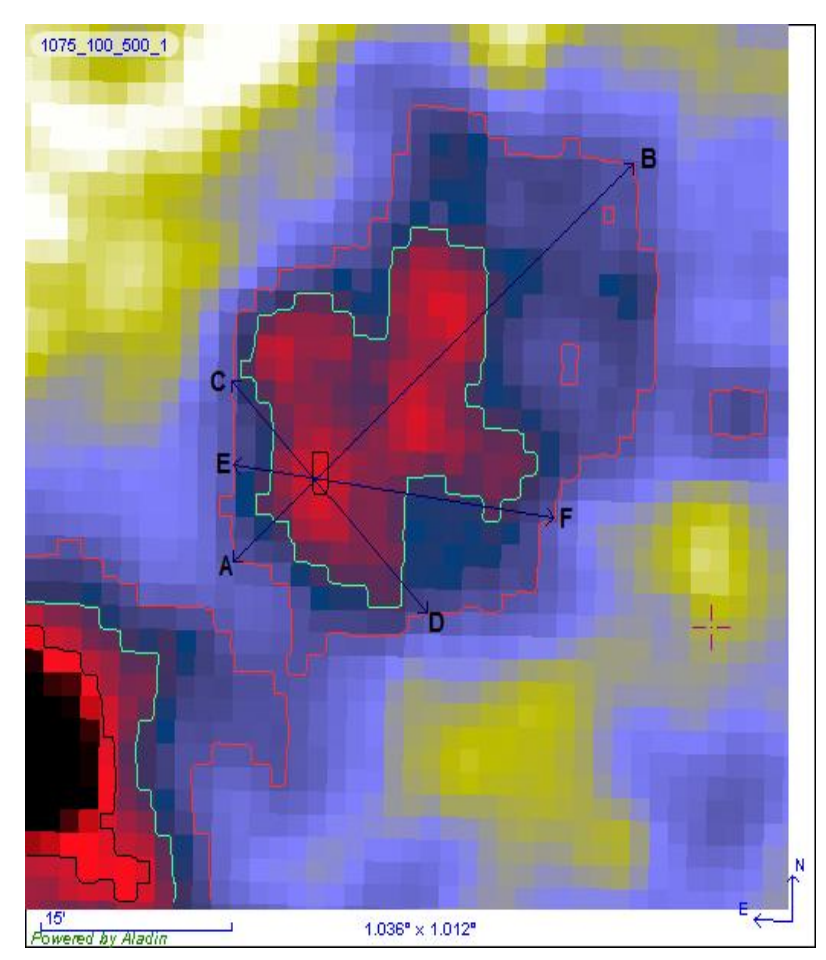

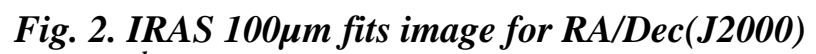
$=18^{h} 57^{m} 26.45^{s} /-10^{\circ} 27^{\prime} 01^{\prime}$ ', here Line $A B$ Representing Major Diameter, CD Minor diameter and $E F$ distance which passes through maximum temperature pixel and minimum flux pixel respectively.

\section{Flux Density Variation}

By using the Aladin v2.5 and Aladin v8.0 softwares, flux density variation of the region of interest is studied. We obtained the graph of flux density variation along the major diameter $(\mathrm{AB})$, minor diameter (CD) and line joining the minimum temperature region and minimum relative flux region $(\mathrm{EF})$ considering cavity as center. As we move from point $\mathrm{B}$ we can see that the decrease in flux slowly at first, after certain distance the flux decrease rapidly to reach the minima point. After reaching minima, the flux increases slightly then decreases to secondary minimum flux. This feature indicates that the mass is expelling by some means from the core of cavity region, this may be due to the strong effect of the prominent pulsar in the interstellar medium. The cause seems to be because of the strong magnetic field developed nearby this region. Therefore, the Gaussian distribution seems to deviate. Best fit polynomial $\left(8^{\text {th }}\right.$ order polynomial, solid line) is shown in figure 3 . 

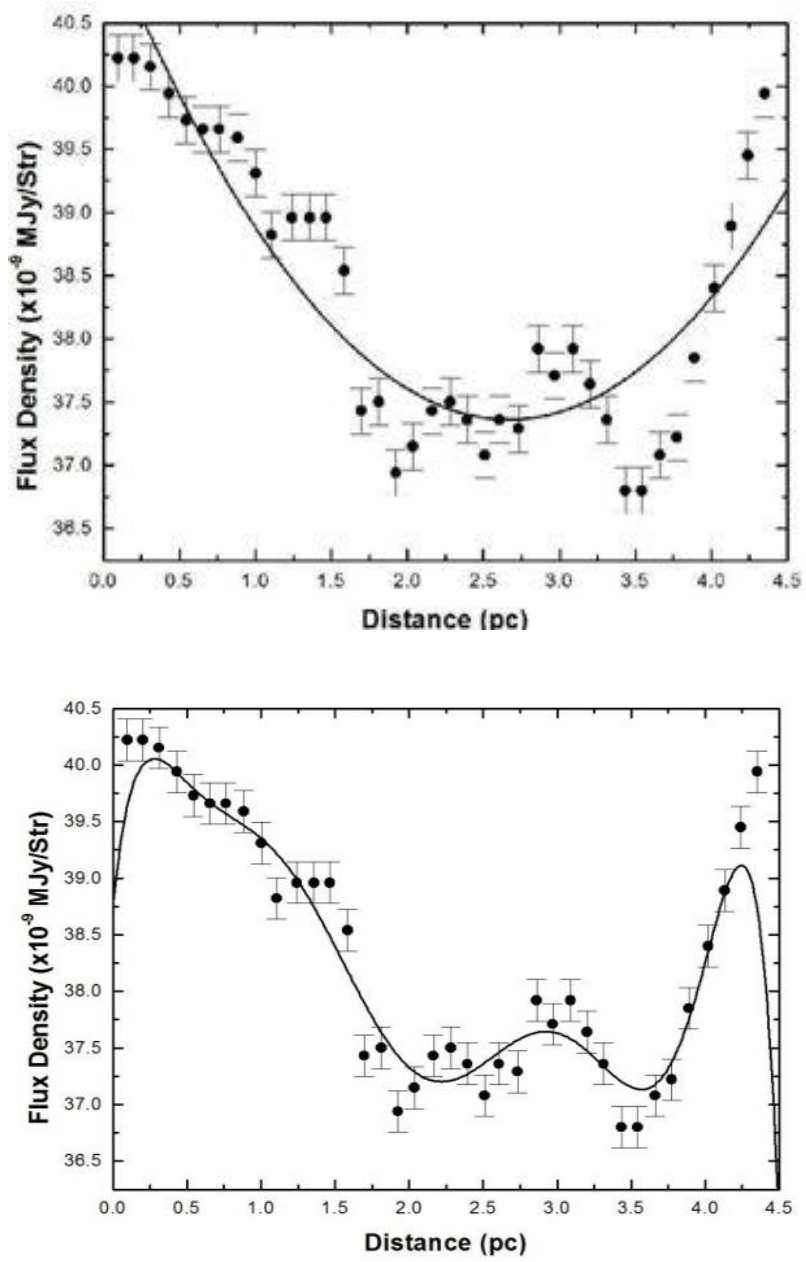

Fig. 3. The distribution of flux density along major diameter $A B$. The solid curve on upper graph represents second order polynomial fit curve whereas lower graph represents the best fit polynomial (8th order polynomial). The solid circles with $\pm \frac{\sigma}{\sqrt{n}}$ error bars represent the standard error of the distribution.

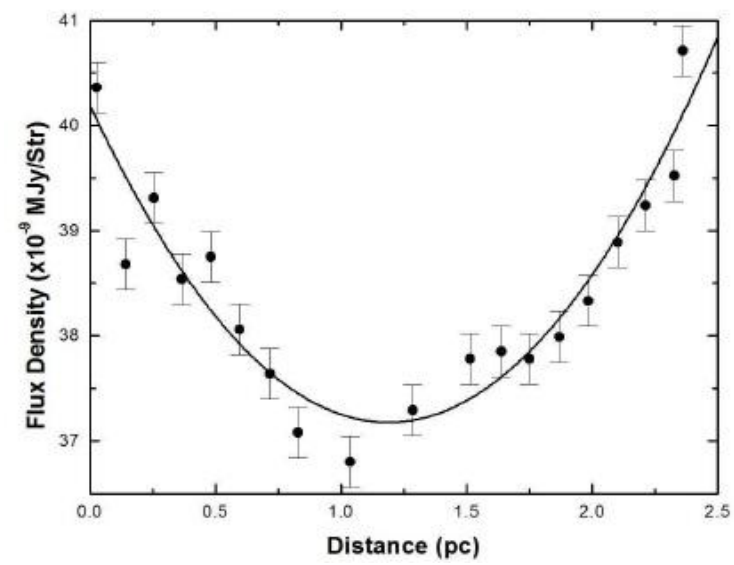

The equation representing the second order polynomial and best fit polynomial (Eighth Order) are given by;

$S_{v}=41.2-2.9 d+0.5 d^{2}$

and,

$\mathrm{S}_{v}=39.7+7.7 \mathrm{~d}-36.3 \mathrm{~d}^{2}+66.8 \mathrm{~d}^{3}-63.2 \mathrm{~d}^{4}+$ $35.52 \mathrm{~d}^{5}-9.2 \mathrm{~d}^{6}+1.3 \mathrm{~d}^{7}-0.1 \mathrm{~d}^{8}$

The Gaussian distribution represents the natural process without any external effect. In other words, we know that the linear form of the Gaussian equation (after taking logarithm) is second order polynomial without constant slope. Equation (11) represents second order polynomial with a small (-2.9) constant slope. Thus a deviation from Gaussian distribution is noticed along the major diameter. A best correlation is found when fitting with eighth order polynomial where slope is even stronger. Hence, the line passing through the major diameter severely deviates from the natural process. The deviation is because of the effect of pulsar wind. Equation (12) is the eighth order polynomial having constant slope (bigger than the second order polynomial), suggesting no closeness between the Gaussian distribution. Hence, it seems that the flux density variation along major diameter is strongly disturbed from the pulsar wind.

The flux density variation with distance along minor diameter (CD) is shown in figure 4. given below. As we search the variation of relative flux density across $\mathrm{CD}$, we find the best fit polynomial of our data is fourth order polynomial.

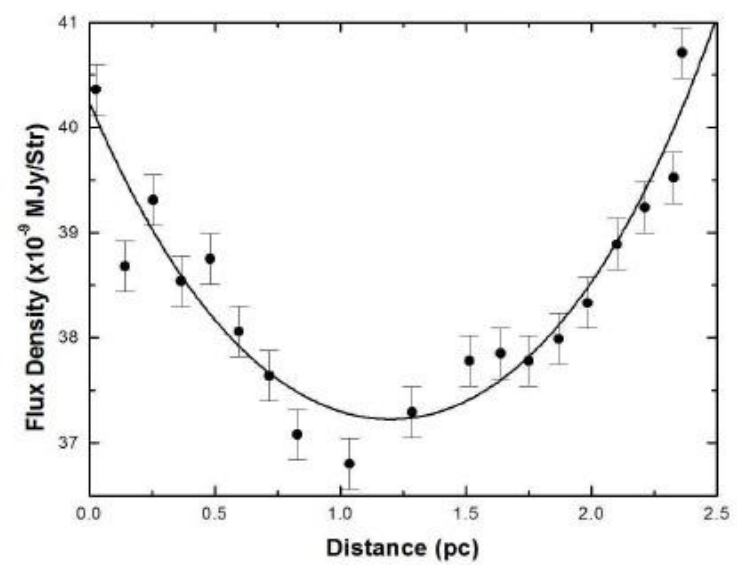

Fig. 4. The distribution of flux density along minor diameter $C D$. The solid curve on left graph represents second order polynomial fit curve whereas right graph represents the best fit polynomial $\left(4^{\text {th }}\right.$ order polynomial). The solid circles with $\pm \frac{\sigma}{\sqrt{n}}$ error bars represent the standard error of the distribution. 
The equation representing the second order polynomial and best fit polynomial $\left(4^{\text {th }}\right.$ Order) are given by;

$S_{v}=40.2-5.1 d+2.1 d^{2}$

And,

$S_{v}=40.2-5.6 d+3.4 d^{2}-0.8 d^{3}+0.2 d^{4}$

Equation (13) represents second order polynomial with a significant negative slope (5.1). Thus a deviation from Gaussian distribution is noticed along the minor diameter. A best correlation is found when fitting with fourth

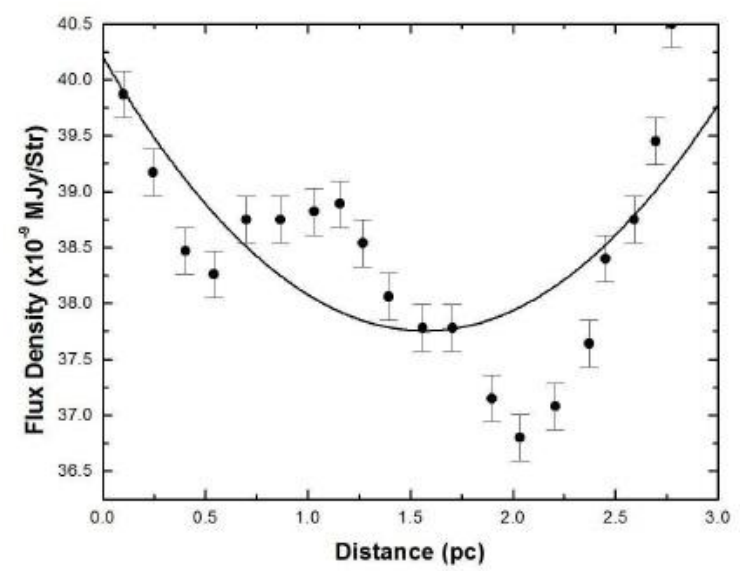

order polynomial where slope is slightly stronger. Hence, the line passing through the minor diameter deviates from the natural process. The deviation is because of the effect of pulsar wind. Equation (14) is the 4th order polynomial having constant slope (-5.6). Hence, it seems that the flux density variation along minor diameter is also disturbed from the pulsar wind but not as strongly as in the major diameter.

As we pointed the location of maximum temperature region we draw a line joining the point of maximum temperature and minimum flux (minima), shown in figure 5. As we search the variation of relative flux density across $\mathrm{EF}$, we find the best fit polynomial of our data is fifth order polynomial.

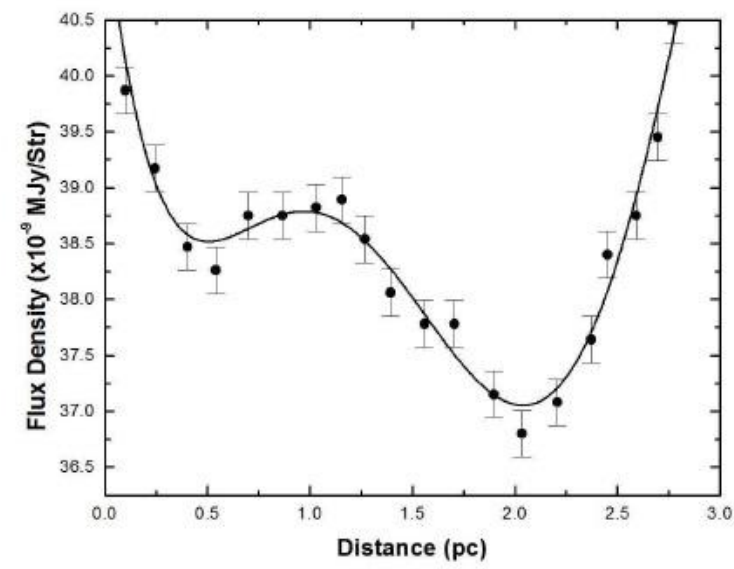

Fig. 5. The distribution of flux density along line passing through minimum flux and maximum temperature,EF. The solid curve on left graph represents second order polynomial fit curve whereas right graph represents the best fit polynomial $\left(5^{\text {th }}\right.$ order polynomial). The solid circles with $\pm \frac{\sigma}{\sqrt{n}}$ error bars represent the standard error of the distribution.

The equation representing the second order polynomial and best fit polynomial $\left(5^{\text {th }}\right.$ Order $)$ are given by;

$\mathrm{S}_{v}=40.2-3.1 \mathrm{~d}+0.9 \mathrm{~d}^{2}$

and,

$S_{v}=41.2-15.2 d+29.6 d^{2}-23.9 d^{3}+8.1 d^{4}-0.9 d^{5}$

From above it is clear that the relative flux density variation is clearly observed first of all decreases slowly after that rapid variation of flux observed up to central region. And with some fluctuations there are asymmetrical behavior observed.

\section{Dust Color Temperature}

Using equation (5), we have calculated dust color temperature of each pixel inside minima lying in the region of interest. We use the IRAS $100 \mu \mathrm{m}$ and $60 \mu \mathrm{m}$ FITS images downloaded from the IRAS server. For the calculation of temperature we choose the value of $\beta=2$ following the explanation given by Dupac. The region with maximum and minimum temperature is found to lie in the range of $29.28 \mathrm{~K}$ to $23.40 \mathrm{~K}$. An offset of about $6 \mathrm{~K}$ suggest that our structure is not independently evolved or the role of discrete point source. This suggests that our region of interest is highly influenced by pulsar wind and its radiation. The average temperature of the dust inside the region of interest is $25.66 \mathrm{~K}$. 
Table 1. The table shows dust color maximum and minimum temperatures and temperature of different region in the innermost contour of the cavity.

\begin{tabular}{|l|l|l|l|}
\hline \multicolumn{1}{|c|}{ Contour } & \multicolumn{1}{|c|}{ R. A. J(2000) } & \multicolumn{1}{c|}{ Dec. J(2000) } & \multicolumn{1}{c|}{$\mathbf{T}_{\mathbf{d}}(\mathbf{K})$} \\
\hline Max. Temperature & $18^{\mathrm{h}} 56^{\mathrm{m}} 41.56^{\mathrm{s}}$ & $10^{\circ} 30^{\prime} 52.9^{\prime \prime}$ & 29.28 \\
\hline Min. Temperature & $18^{\mathrm{h}} 57^{\mathrm{m}} 16.32^{\mathrm{s}}$ & $10^{\circ} 11^{\prime} 22.4^{\prime \prime}$ & 23.40 \\
\hline Innermost contour & $18^{\mathrm{h}} 57^{\mathrm{m}} 11.29^{\mathrm{s}}$ & $10^{\circ} 14^{\prime} 11.6^{\prime \prime}$ & 24.17 \\
& $18^{\mathrm{h}} 57^{\mathrm{m}} 48.51^{\mathrm{s}}$ & $10^{\circ} 33^{\prime} 37.1^{\prime \prime}$ & 24.36 \\
& $18^{\mathrm{h}} 57^{\mathrm{m}} 41.15^{\mathrm{s}}$ & $10^{\circ} 32^{\prime} 06.2^{\prime \prime}$ & 24.69 \\
& $18^{\mathrm{h}} 57^{\mathrm{m}} 11.35^{\mathrm{s}}$ & $10^{\circ} 24^{\prime} 38.7^{\prime \prime}$ & 24.86 \\
& $18^{\mathrm{h}} 58^{\mathrm{m}} 12.06^{\mathrm{s}}$ & $10^{\circ} 21^{\prime} 31.5^{\prime \prime}$ & 24.93 \\
& $18^{\mathrm{h}} 57^{\mathrm{m}} 11.31^{\mathrm{s}}$ & $10^{\circ} 23^{\prime} 08.5^{\prime \prime}$ & 25.67 \\
& $18^{\mathrm{h}} 57^{\mathrm{m}} 17.16^{\mathrm{s}}$ & $10^{\circ} 23^{\prime} 38.7^{\prime \prime}$ & 26.05 \\
\hline
\end{tabular}

\section{Dust Mass}

The flux density and the distance are used to estimate the dust mass in our region of interest using IRAS image. The infrared flux is obtained from Groningen IRAS server available at official website of Sky View virtual observatory. By using the average of the maximum and minimum temperature of each maxima summing up of the flux density of each pixel inside the region, the value of average temperature and the total flux density for all the maxima can be known. For the calculation of the mass we need the distance to the region of interest. The distance is recommended to be $-375 \mathrm{pc}$ and we use this distance in our calculation. The background correction is made by subtracting the background flux density from the flux density measured directly from image.

We have calculated the dust mass of the structure enclosed by each contour using equation (9). The separate mass of the structure enclosed by the each contour is tabulated below;

Table 2. The table shows the Sum, Average, and excess mass per Pixel of region of Interest.

\begin{tabular}{|l|l|l|l|l|}
\hline Contours & Mass $(\mathbf{k g})$ & $\mathbf{M}\left(\mathbf{M}_{\mathbf{\Theta}}\right)$ & Average Mass $(\mathbf{k g})$ & Excess Mass per pixel (kg) \\
\hline Inner & $3.19 \times 10^{26}$ & 0.000161 & $3.85 \times 10^{24}$ & $2.52 \times 10^{24}$ \\
\hline Middle & $5.21 \times 10^{26}$ & 0.000263 & $6.04 \times 10^{24}$ & \\
\hline Outer & $1.71 \times 10^{27}$ & 0.000864 & $8.64 \times 10^{24}$ & \\
\hline Total & $2.55 \times 10^{27}$ & 0.0013 & & \\
\hline
\end{tabular}

In the table, first column contains different contour levels, second column contains dust mass of corresponding contours, third column gives the dust mass of contour with compared to solar mass $\mathbf{M}_{\boldsymbol{\Theta}}$, the fourth column represents the average dust mass and the last column represents the excess mass per pixel. Excess Mass (Mass deficit) per pixel is calculated by the difference of average dust masses of smaller circle from the bigger circle which is divided by the total number of pixels, called the outflow mass, as shown in figure 6 . 

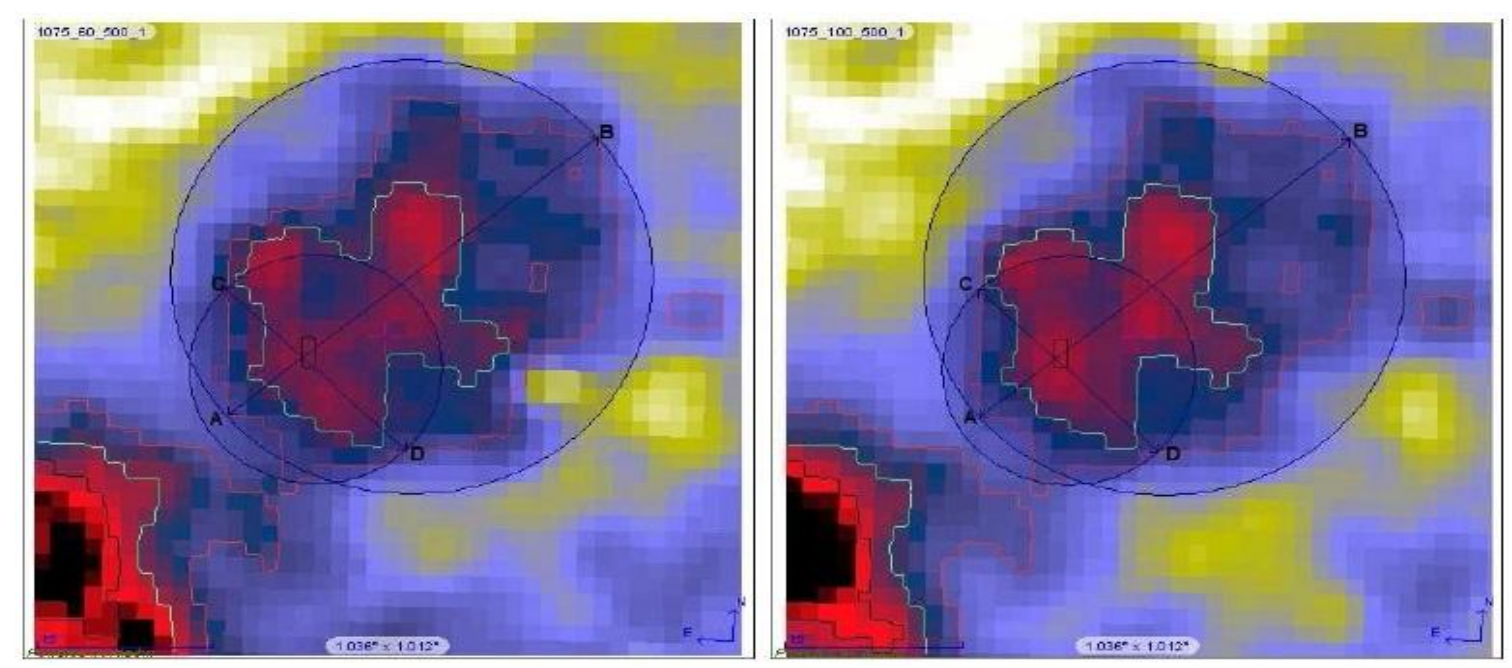

Fig. 6. Line AB representing major diameter CD representing minor diameter. Here the circles drown are assigned as smaller and bigger circle used for observation of outflow nature of Pulsar wind.

\section{Energy Estimation for Outflow Mass.}

The energy is estimated by using the classical mass energy relation that is required to pull the excess mass by the pulsar and is given by

$E=\frac{1}{2} M_{\text {deficit }} u_{\text {out }}^{2}$

$\mathrm{M}_{\text {deficit }}$ is the mass deficit per pixel which is calculated to be $2.52 \times 10^{24} \mathrm{~kg}$ and the velocity of the pulsar wind is $2000 \mathrm{~km} / \mathrm{sec}$, we got the required energy for the structure formation as $\mathrm{E}=5.04 \times 10^{36} \mathrm{~J}$.

\section{CONCLUSION}

This research work was aimed to contribute to our understanding of the interaction between interstellar medium and wind emitted by pulsar star. The size of the structure is $4.46 \mathrm{pc} \times 2.36 \mathrm{pc}$ where, major and minor diameters are found to be $4.46 \mathrm{pc}$ and $2.36 \mathrm{pc}$ respectively. The minimum flux region is found to be located at center of lower part, at R.A. $(\mathrm{J} 2000)=18^{\mathrm{h}} 57^{\mathrm{m}} 53.81^{\mathrm{s}}, \quad$ Dec $(\mathrm{J} 2000)=$ $-10^{0} 29^{\prime} 05.1$ ”. A study of distance versus flux density variation shows a prominent cavity structure at $100 \mu \mathrm{m}$ image and distribution of flux density variation along major diameter of the structure shows that it is strongly deviated from Gaussian distribution which is due to pulsar wind for the formation of that cavity whereas along minor and line joining minimum flux and maximum temperature, it is not so prominent. The region with maximum and minimum temperature is found to lie in the range of $29.28 \mathrm{~K}$ to $23.40 \mathrm{~K}$. An offset temperature of about $6 \mathrm{~K}$ suggests that our structure is not independently evolved. This offset of temperature greater than $5 \mathrm{~K}$ suggests that the cavity is either very dip or might be formed due to various processes such as interstellar bubble formation, dust and grain formation etc. The total mass of the dust in the structure is found about $2.55 \times 10^{27} \mathrm{~kg}\left(0.0013 \mathrm{M}_{\odot}\right)$. The mass of big circle and small circle are found to be $6.12 \times 10^{27} \mathrm{~kg}$ and $1.33 \times 10^{27} \mathrm{~kg}$ respectively and hence mass deficit per pixel is found to be $2.52 \times 10^{24} \mathrm{~kg}$. The energy of the pulsar to expel the excess mass is found to be $5.04 \times 10^{36} \mathrm{~J}$.

In this way we have studied the Interaction between ISM and Pulsar Wind from which we obtained lots of physical properties and parameters using different techniques with the help of different software. Hence from above observation it is concluded that asymmetrical phenomena was observed.

\section{ACKNOWLEDGEMENTS}

We are indebted to Prof. Ronald Weinberger of Innsbruck University, Innsbruck, Austria, for providing substantial data. This research has made use of NASA/IPAC Extragalactic Database (NED) which is operated by the Jet Propulsion Laboratory, California Institute of Technology, under contract with the National Aeronautics and Space Administration. One of the author (AKJ) acknowledges CDP, T. U. for providing substantial support for his $\mathrm{Ph}$. D. degree. 


\section{REFERENCES}

Becker, W. (2009). Neutron Stars and Pulsars. Springe.U.S.A. Chapter 2, pp.203.

Beichman, C. A.; Neugebauer, G.; Habing, H. J.; Clegg, P. E. and Chester, T. J. (1988). Infrared Astronomical Satellite (IRAS) Catalogues and Atlases I: Explanatory Supplement. US Government Printing Office, Washington.

Bhattacharya, A. B.; Joardar, S. and Bhattacharya, R. (2008). Astronomy \& Astrophysics, Infinity Science Press, India, chapter 19, pp.273.

Dupac, X.; Bernard, J. P.; Boudet, N.; Giard, M.; Lamarre, J. M.; Mny, C. et al. (2003). Inverse Temperature Dependence of the dust submillimeter spectral index. Astronomy and Astrophysics, 404: L11-L15.

Giles, A. B. (1982). A Powerful Method for Star Counting in the Infrared. Monthly Notices of the Royal Astronomical Society, 199: 483491.

Hildebrand, R. H. (1983). The Determination of Cloud Masses and Dust Characteristics from
Submillimeter Theral Emission. Q. Journal of Royal Astronomical Society, 24: 267-282.

Johnston, S.; Wex, N.; Nicastro, L.; Manchester, N. and Lyne, A. G. (2001). The 1997 Periastron Passage of the Binary Pulsar PSRB1259-63. Monthly Notice of Royal Astronomical Society, 326: 643-648.

Karttunen, H.; Kro“oger, P.; Oja, H.; Poutanen, M. and Donner, K. J. (2007). Fundamental Astronomy, $5^{\text {th }}$ edition, Springer Berlin Heidelberg, USA.

Pacini, F. (1968). Rotating Neutron stars, Pulsars and Supernova remnants. Nature, 219: 145146.

Schnee, S. L.; Ridge, N. A.; Goodman, A. A. and Jason, G. L. (2005). A Complete Look at the Use of IRAS Emission Maps to Estimate Extinction and Dust Temperature. Astrophysical Journal, 634: 442-450.

Suh, K. W. and Kwon, Y. J. (2009). A Catalog of AGB Stars in IRAS psc. Journal of the Korean Astronomical Society, 42: 81-91.

http://simbad.u-strasbg.fr/simbad/(SIMBAD, 2014). 